01

\title{
Поверхностные волны на границе фоторефрактивного кристалла и среды с положительной керровской нелинейностью
}

\author{
(C) C.E. Савотченко \\ Белгородский государственный технологический университет им. В.Г. Шухова \\ Белгород, Россия \\ E-mail: savotchenkose@mail.ru
}

Поступила в Редакцию 19 октября 2019 г.

В окончательной редакции 19 октября 2019 г.

Принята к публикации 25 октября 2019 г.

\begin{abstract}
Описаны типы нелинейных поверхностных волн необыкновенной поляризации, возникающие на границе раздела фоторефрактивного кристалла и среды с положительной керровской нелинейностью. Показано, что в такой системе могут существовать нелинейные поверхностные волны несимметричного профиля двух типов. Волны первого типа затухают при удалении от границы раздела без осцилляций как в глубину фоторефрактивного кристалла, так и керровского, а волны второго типа затухают в глубину фоторефрактивного кристалла с осцилляциями. Получены дисперсионные соотношения и указаны условия существования всех описанных типов волн в зависимости от оптических характеристик кристаллов.
\end{abstract}

Ключевые слова: граница раздела сред, фоторефрактивный кристалл, оптическая нелинейная среда, нелинейные поверхностные волны.

DOI: 10.21883 /FTT.2020.06.49345.624

\section{1. Введение}

Существует множество теоретических работ, посвященных описанию поверхностных электромагнитных волн (плазмонов, поляритонов) на границах различных сред [1-7]. Особое значение имеют исследования свойств поверхностных волн в различных нелинейных оптических средах, таких как фоторефрактивные кристаллы [8-11] и среды с эффектом Керра [12]. Однако особенности распространения нелинейных поверхностных волн на границах сред, в которых нелинейные эффекты обусловлены различными механизмами перераспределения плотности зарядов и индицированного ими поля, остаются мало изученными. Поэтому требуют детального анализа многие практически важные аспекты, в частности, закономерности формирования нелинейных поверхностных волн с несимметричным профилем, возникающих вблизи границ между фоторефрактивными кристаллами и другими нелинейными оптическими средами.

В большинстве существующих теоретических работ рассматривается локализация возбуждений электромагнитного поля вблизи границ раздела сред с одинаковыми по физической природе формами нелинейности (наиболее часто встречающийся случай - контакт двух сред с керровской нелинейностью [13-18]), либо на границе раздела линейной и нелинейной сред [19-22].

В настоящей работе предлагается теоретичное описание новых типов нелинейных локализованных состояний, соответствующих поверхностным волнам на границе двух оптических сред с различными по физической природе формами нелинейности: фоторефрактивно- го кристалла и кристалла с керровской нелинейностью. Очевидно, что такие поверхностные волны будут иметь несимметричный профиль относительно границы раздела сред.

\section{2. Формулировка модели}

Рассмотрим контакт одноосного фоторефрактивного кристалла с диффузионным механизмом формирования нелинейности и одноосного кристалла с керровской нелинейностью (далее будем для краткости называть его керровским кристаллом) в отсутствии приложенного внешнего поля. Границу раздела кристаллов будем считать настолько тонкой, что можно будет пренебречь оптическими эффектами внутри нее.

Будем изучать Р-поляризованные нелинейные поверхностные волны, для которых $E_{y}=0, H_{x}=H_{z}=0$, т.е. волны с необыкновенной поляризацией (ТМ-волны). Так как будет рассматриваться скользящее распространение светового пучка, то можно пренебречь анизотропией показателя преломления и использовать одноосное приближение.

Пусть полярная ось фоторефрактивного кристалла направлена вдоль оси $x$. ТМ-волна распространяется вдоль оси $z$. Граница раздела между фоторефрактивным и керровским кристаллами расположена в плоскости $x=0$. Фоторефрактивный кристалл занимает полупространство $x>0$, а керровский - полупространство $x<0$.

Будем рассматривать только стационарное распределение поля поверхностной волны. Из системы уравнений Максвелла в рассматриваемом случае получается уравнение для отличной от нуля компоненты вектора 
магнитного поля

$$
\Delta H_{y}+k^{2}(x) H_{y}=0
$$

где $\Delta$ - двумерный оператор Лапласа по координатам $x$ и $z$,

$$
k(x)=k_{0}\left\{n_{j 0}(x)+\Delta n_{j}(x)\right\},
$$

$k_{0}=2 \pi / \lambda_{0}, \lambda_{0}$ - длина волны света в вакууме, $n_{j 0}-$ невозмущенные показатели преломления, $\Delta n_{j}-$ нелинейные добавки к ним, которые считаются малыми $\left(\Delta n_{j} \ll n_{j 0}\right), j=1,2$. Здесь и далее значение индекса $j=1$ соответствует величине, характеризующей фоторефрактивный кристалл в области $x>0$, а значение индекса $j=2$ соответствует величине, характеризующей керровский кристалл в области $x<0$.

Нелинейная добавка к показателю преломления фоторефрактивного кристалла формируется в результате диффузионного механизма нелинейности [23]. Если пренебречь темновой интенсивностью по сравнению с интенсивностью поверхностной волны, то нелинейную добавку к показателю преломления фоторефрактивного кристалла можно представить в виде $[9,10,23]$ :

$$
\Delta n_{1}(x)=\frac{1}{2} n_{10}^{3} r_{e f f} \frac{k_{B} T}{e} \frac{I_{1}^{\prime}}{I_{1}}
$$

где штрихи здесь и далее означают производные по координате $x, r_{e f f}$ - эффективный электрооптический коэффициент, $k_{\mathrm{B}}$ - константа Больцмана, $T-$ температура, $e-$ модуль заряда электрона, $I_{j} \propto\left|H_{j}\right|^{2}-$ интенсивность светового пучка в поверхностной волне.

Нелинейная добавка к показателю преломления керровского кристалла пропорциональна интенсивности: $\Delta n_{2}(x) \propto I_{2}$. Для нее будем использовать выражение в виде: $\Delta n_{2}(x)=\alpha\left|H_{2}\right|^{2}, \alpha-$ коэффициент керровской нелинейности. В данной работе он считается постоянным и положительным, что соответствует керровской среде с фокусировкой.

Предполагая, что установившееся распределение поля в распространяющейся вдоль оси $\mathrm{Z}$ волне представимо в виде

$$
H_{y}(x, z)=H_{j}(x) e^{i \beta k_{0} z},
$$

где $\beta$ - константа распространения, в рассматриваемом приближении с учетом малости темновой интенсивности по сравнению с интенсивностью поверхностной волны и малости невозмущенных показателей преломления по сравнению с нелинейными добавками к ним, из (1) можно получить уравнения

$$
\begin{gathered}
H_{1}^{\prime \prime}+\mu H_{1}^{\prime}+\left(n_{10}^{2}-\beta^{2}\right) k_{0}^{2} H_{1}=0, \\
H_{2}^{\prime \prime}+\left(n_{20}^{2}-\beta^{2}\right) k_{0}^{2} H_{2}+g\left|H_{2}\right|^{2} H_{2}=0,
\end{gathered}
$$

где обозначили $\mu=2 k_{0}^{2} n_{10}^{4} r_{e f f} k_{B} T / e-$ коэффициент затухания волны в фоторефрактивном кристалле, $g=2 \alpha k_{0}^{2} n_{20}-$ коэффициент нелинейности в керровском кристалле (в данной работе он считается положительной величиной).
Из условия непрерывности тангенциальных составляющих полей на границе кристаллов следуют граничные условия

$$
\begin{aligned}
& H_{1}(0)=H_{2}(0)=H_{0}, \\
& \frac{1}{\varepsilon_{1}} H_{1}^{\prime}(0)=\frac{1}{\varepsilon_{2}} H_{2}^{\prime}(0),
\end{aligned}
$$

где $H_{0}-$ амплитуда поля на границе раздела, $\varepsilon_{j} \propto n_{j 0}^{2}-$ линейные (невозмущенные) части диэлектрических проницаемостей фоторефрактивного и керровского кристаллов соответственно.

Таким образом, математическая формулировка модели для описания нелинейных поверхностных волн на границе фоторефрактивного и керровского кристаллов сводится к уравнениям (2) и (3) с граничными условиями (4) и (5).

\section{3. Нелинейные поверхностные волны}

Уравнение (2) имеет два типа исчезающих на бесконечности решений в зависимости от соотношения между значениями константы распространения, коэффициента затухания и невозмущенного показателя преломления в фоторефрактивном кристалле. Амплитуда волны первого типа затухает без осцилляций при удалении от границы раздела вглубь фоторефрактивного кристалла, а второго - осциллирующим образом [24].

Решения нелинейного уравнения (3) определяются знаком коэффициента нелинейности $g$ и знаком разности $n_{20}^{2}-\beta^{2}$. В зависимости от их комбинаций возникает несколько типов нелинейных поверхностных волн несимметричного профиля.

В настоящей работе будем рассматривать керровскую среду только с положительным коэффициентом нелинейности.

\section{1. Затухающие без осцилляций волны}

При $g>0$ и $\max \left\{n_{20}, \sqrt{n_{10}^{2}-\mu^{2} / 4 k_{0}^{2}}\right\}<\beta<n_{10}$ решение уравнений (2) и (3) представимы в виде

$$
\begin{gathered}
H_{1}(x)=e^{-\mu x / 2}\left(A e^{v x}+B e^{v x}\right), \\
H_{2}(x)=\sqrt{\frac{2}{g}} \frac{q}{\operatorname{ch} q\left(x-x_{0}\right)} .
\end{gathered}
$$

где

$$
\begin{gathered}
v^{2}=\frac{1}{4}\left\{\mu^{2}-4 k_{0}^{2}\left(n_{10}^{2}-\beta^{2}\right)\right\}, \\
q^{2}=k_{0}^{2}\left(\beta^{2}-n_{20}^{2}\right) .
\end{gathered}
$$

Подстановка решений (6) и (7) в граничные условия (4) и (5) приводит к выражениям для параметров поля в фоторефрактивном кристалле

$$
\begin{aligned}
A & =\frac{q}{v \sqrt{2 g} \operatorname{ch} q x_{0}}\left(\frac{\varepsilon_{1}}{\varepsilon_{2}} q \text { th } q x_{0}+\frac{\mu}{2}+v\right), \\
B & =-\frac{q}{v \sqrt{2 g} \operatorname{ch} q x_{0}}\left(\frac{\varepsilon_{1}}{\varepsilon_{2}} q \text { th } q x_{0}+\frac{\mu}{2}-v\right) .
\end{aligned}
$$


Нелинейная поверхностная волна с неосциллирующим профилем (6) может затухать в глубину фоторефрактивного кристалла немонотонно или монотонно. Монотонное затухания волны в глубь фоторефрактивного кристалла может происходить в двух случаях:

1) $A=0$ и тогда $H_{0}=B$ или

2) $B=0$ и тогда $H_{0}=A$.

Тогда из (10) и (11) получается

$$
v=\mp\left(\frac{\varepsilon_{1}}{\varepsilon_{2}} q \text { th } q x_{0}+\frac{\mu}{2}\right),
$$

где выбирается знак, „-“ для $A=0$ и , $+{ }^{\text {“ }}$ для $B=0$. В этих случаях распределение поля (6) в монотонной затухающей поверхностной волне в фоторефрактивном кристалле принимает вид

$$
H_{1}(x)=H_{0} e^{-\gamma x},
$$

где амплитуда поля на границе раздела

$$
H_{0}=\sqrt{2} q / \sqrt{g} \operatorname{ch} q x_{0},
$$

коэффициент затухания

$$
\gamma=-\varepsilon_{1} q \text { th } q x_{0} / \varepsilon_{2}
$$

Для положительности коэффициента затухания в монотонной убывающей волне должно быть $x_{0}<0$. Такая монотонно убывающая волна существует при фиксированной связи константы распространения, коэффициентов преломления и других физических характеристик фоторефрактивного и керровского кристаллов, определяемой выражением (12).

В отличие от такой монотонной убывающей поверхностной волны, поверхностная волна, в котором поле в фоторефрактивном кристалле описывается выражением (6), может иметь экстремум на расстоянии $x_{m}=v^{-1} \ln I$, где

$$
I=\frac{(v+\mu)\left(v-\mu / 2-\left(\varepsilon_{1} / \varepsilon_{2}\right) g \text { th } q x_{0}\right)}{(v-\mu)\left(v+\mu / 2+\left(\varepsilon_{1} / \varepsilon_{2}\right) g \text { th } q x_{0}\right)} .
$$

Экстремальное значение амплитуды поля, достигаемое на расстоянии хm от границы раздела в глубину фоторефрактивного кристалла, можно оценить по формуле

$$
H_{m}=\frac{q I^{-(\nu+\mu / 2)}}{v \sqrt{2 g} \operatorname{ch} q x_{0}}\left(\frac{2 \varepsilon_{1} q\left(v^{2}+\mu^{2} / 4\right) \text { th } q x_{0}}{\varepsilon_{2}\left(v^{2}-\mu^{2} / 4\right)}-\mu\right) .
$$

\section{2. Затухающие с осцилляциями волны}

Если теперь считать, что $n_{20}<\beta<\sqrt{n_{10}^{2}-\mu^{2} / 4 k_{0}^{2}}$, то при $g>0$ решение уравнения (3) остается в виде (7), а решение уравнения (2) примет вид

$$
\begin{aligned}
& H_{1}(x)=H_{0} e^{-\mu x / 2} \cos (p x+\varphi) / \cos \varphi, \\
& p^{2}=-v^{2}=\frac{1}{4}\left\{4 k_{0}^{2}\left(n_{10}^{2}-\beta^{2}\right)-\mu^{2}\right\} .
\end{aligned}
$$

Распределение поля (14) в поверхностной волне затухает с осцилляциями при удалении от границы раздела в глубину фоторефрактивного кристалла. Характерный период осцилляций поля: $\Lambda=2 \pi / p$, глубина проникновения в фоторефрактивный кристалл: $l=2 / \mu$. Затухание поля в глубину керровского кристалла по-прежнему происходит без осцилляций.

Подстановка решений (7) и (14) в граничные условия (4) и (5) приводит к амплитуде поля $H_{0}$ в фоторефрактивном кристалле в виде (13), а также к дисперсионному соотношению

$$
p \operatorname{tg} \varphi=-\left(\frac{\varepsilon_{1}}{\varepsilon_{2}} q \text { th } q x_{0}+\frac{\mu}{2}\right) .
$$

Дисперсионное соотношение (16), определяющее зависимость константы распространения от коэффициентов преломления и других физических характеристик фоторефрактивного и керровского кристаллов, можно проанализировать в различных предельный случаях.

В частности, при $\varphi=0$ и $q x_{0} \ll 1$ из (16) можно получить в явном виде закон дисперсии

$$
\beta^{2}\left(x_{0}\right)=n_{20}^{2}-\frac{\mu \varepsilon_{2}}{2 \varepsilon_{1} x_{0} k_{0}^{2}} \text {. }
$$

Волна с законом дисперсии (17) существует при $x_{0}<0$.

При $x_{0}=0$ и меняющемся $\varphi$ из (16) получается закон дисперсии в виде

$$
\beta^{2}(\varphi)=n_{10}^{2}-\frac{\mu^{2}}{4 k_{0}^{2}}\left(1+\operatorname{ctg}^{2} \varphi\right)
$$

Условие существования такой волны с законом дисперсии (18) определятся неравенством: $\operatorname{ctg}^{2} \varphi<4 k_{0}^{2} n_{10}^{2} / \mu^{2}-1$.

При $q x_{0} \ll 1$ и меняющемся $\varphi$ из (16) получается закон дисперсии в виде

$$
\begin{gathered}
\beta^{2}(\varphi)=n_{10}^{2}-\frac{\mu^{2}}{4 k_{0}^{2}}-\left(\frac{\varepsilon_{2} \operatorname{tg} \varphi}{2 \varepsilon_{1} k_{0} x_{0}}\right)^{2}\left\{1 \pm D^{1 / 2}\right\}, \\
D=1-f \operatorname{ctg}^{2} \varphi, \\
f=\frac{4 \varepsilon_{1} x_{0}}{\varepsilon_{2}}\left\{\frac{\varepsilon_{1} x_{0}}{\varepsilon_{2}}\left[\frac{\mu^{2}}{4}-k_{0}^{2}\left(n_{10}^{2}-n_{20}^{2}\right)\right]-\frac{\mu}{2}\right\} .
\end{gathered}
$$

Условие существования такой волны с законом дисперсии (20) определятся неравенством: $\operatorname{tg}^{2} \varphi>f$.

При меняющемся $\varphi$ в основном приближении константа распространения зависит от квадрата коэффициента затухания волны в фоторефрактивном кристалле. От коэффициента керровской нелинейности зависит только амплитуда поверхностной волны. 


\section{4. Зависимость константы распространения от амплитуды поля}

В теории нелинейных колебаний часто такие характеристики как, например, частота, выражаются через амплитуду. Для нелинейных поверхностных волн имеет смысл проанализировать зависимость частоты константы распространения от амплитуды поля на границе раздела кристаллов. В п. 3 проведен анализ поведения константы распространения от свободного (управляющего) параметра $x_{0}$, характеризующего положение „центра солитона“ в керровском кристалле. Далее в качестве управляющего параметра выберем величину $H_{0}$, определяемую выражением (13). В результате удается найти точные решения дисперсионных уравнений в явном аналитическом виде.

\section{1. Зависимость константы распространения от амплитуды монотонно затухающих волн}

Если исключить из (12) $x_{0}$ с помощью (13), то получится дисперсионное уравнение

$$
v=\mp\left(\frac{\varepsilon_{1}}{\varepsilon_{2}} \sqrt{q^{2}-\frac{g H_{0}^{2}}{2}}+\frac{\mu}{2}\right),
$$

точное решение которого позволяет при использовании (8) и (9) получить зависимость константы распространения от амплитуды в виде

$$
\begin{gathered}
\beta^{2}\left(H_{0}\right)=n_{20}^{2}+\frac{1}{2 \delta \varepsilon^{2} k_{0}^{2}}\left\{\left(\frac{\mu \varepsilon_{1}}{\varepsilon_{2}}\right)^{2}-2 \delta \varepsilon d_{0} \pm D_{0}^{1 / 2}\right\}, \\
\delta \varepsilon=1-\left(\frac{\varepsilon_{1}}{\varepsilon_{2}}\right)^{2}, \quad d_{0}=\left(\frac{\varepsilon_{1}}{\varepsilon_{2}}\right)^{2} \frac{g H_{0}^{2}}{2}-k_{0}^{2}\left(n_{10}^{2}-n_{20}^{2}\right), \\
D_{0}=\left\{\left(\frac{\mu \varepsilon_{1}}{\varepsilon_{2}}\right)^{2}-2 \delta \varepsilon d_{0}\right\}^{2}-4 \delta \varepsilon^{2}\left\{d_{0}^{2}+\left(\frac{\mu \varepsilon_{1}}{\varepsilon_{2}}\right)^{2} \frac{g H_{0}^{2}}{2}\right\} .
\end{gathered}
$$

Из (22) следует, что в случае малоамплитудных колебаний в основном приближении квадрат константы распространения зависит линейно от квадрата амплитуды: $\beta^{2}\left(H_{0}\right)=n_{20}^{2}+a H_{0}^{2}$, где $a$ определяется параметрами кристаллов и не зависит от амплитуды $H_{0}$.

Если амплитуда на границе раздела кристаллов связана с оптическими характеристиками кристаллов специальным соотношением (получаемым из условия $\left.\left(\mu \varepsilon_{1} / \varepsilon_{2}\right)^{2}=2 \delta \varepsilon d_{0}\right)$ :

$$
H_{0}^{2}=\frac{1}{g}\left\{\frac{\mu^{2}}{\delta \varepsilon}+2 k_{0}^{2}\left(n_{10}^{2}-n_{20}^{2}\right)\left(\frac{\varepsilon_{2}}{\varepsilon_{1}}\right)^{2}\right\},
$$

то из (22) следует зависимость константы распространения поверхностной волны

$$
\begin{aligned}
\beta^{2}\left(H_{0}\right)= & n_{20}^{2}+\frac{1}{k_{0}^{2}}\left\{-\frac{1}{\delta \varepsilon}\left[\left(\frac{\mu \varepsilon_{1}}{\varepsilon_{2}}\right)^{2} \frac{g H_{0}^{2}}{2}\right.\right. \\
& \left.\left.+\left(\left(\frac{\varepsilon_{1}}{\varepsilon_{2}}\right)^{2} \frac{g H_{0}^{2}}{2}-k_{0}^{2}\left(n_{10}^{2}-n_{20}^{2}\right)\right)^{2}\right]\right\}^{1 / 2},
\end{aligned}
$$

куда подставляется (23). Для существования такой поверхностной волны достаточно того, чтобы линейный показатель преломления фоторефрактивного кристалла был больше линейного показателя преломления керровского кристалла.

В случае малости $\delta \varepsilon \ll 1$, что соответствует тому, что значения линейных показателей преломления фоторефрактивного керровского кристаллов близки, из (21) можно получить константу распространения в виде

$$
\begin{aligned}
& \beta^{2}\left(H_{0}\right)=n_{20}^{2}+\frac{1}{k_{0}^{2}} \\
& \times\left\{\frac{g H_{0}^{2}}{2}+\left(\frac{\varepsilon_{2}}{\mu \varepsilon_{1}}\right)\left(\left(\frac{\varepsilon_{1}}{\varepsilon_{2}}\right)^{2} \frac{g H_{0}^{2}}{2}-k_{0}^{2}\left(n_{10}^{2}-n_{20}^{2}\right)\right)^{2}\right\} .
\end{aligned}
$$

Если линейный показатель преломления фоторефрактивного кристалла много больше линейного показателя преломления керровского кристалла, из (21) можно получить константу распространения в виде

$$
\beta^{2}\left(H_{0}\right)=n_{20}^{2}+\frac{g H_{0}^{2}}{2 k_{0}^{2}} \text {. }
$$

В таком предельном случае $\left(n_{20} \ll n_{10}\right)$ значение константы распространения при малых амплитудах близко к линейному показателю преломления керровского кристалла, а ее квадрат линейно зависит от квадрата амплитуды поля на границе раздела кристаллов.

Следует отметить, что зависимости (25) и (26) являются приближенными решениями дисперсионного уравнения (21) в отличие от зависимости (22) и ее частных случаев (23) и (24), которые являются точными решениями дисперсионного уравнения (21).

\section{2. Зависимость константы распространения от амплитуды затухающих с осцилляциями волн}

Если исключить $x_{0}$ из (16) с помощью (13), то получится дисперсионное уравнение

$$
p \operatorname{tg} \varphi=-\left(\frac{\varepsilon_{1}}{\varepsilon_{2}} \sqrt{q^{2}-\frac{g H_{0}^{2}}{2}}+\frac{\mu}{2}\right),
$$


точное решение которого позволяет при использовании (9) и (15) получить зависимость константы распространения от амплитуды при меняющемся $\varphi$ в виде

$$
\begin{gathered}
\beta^{2}\left(H_{0}, \varphi\right)=n_{10}^{2}-\frac{1}{2 \delta \varepsilon_{\varphi} k_{0}^{2}}\left\{\delta \varepsilon_{\varphi} d_{\varphi}-\left(\frac{\mu \varepsilon_{1}}{\varepsilon_{2}}\right)^{2} \pm D_{\varphi}^{1 / 2}\right\}, \\
\delta \varepsilon_{\varphi}=\frac{\varepsilon_{1}}{\varepsilon_{2}}+\operatorname{tg}^{2} \varphi, \\
b_{\varphi}=\left(\frac{\varepsilon_{1}}{\varepsilon_{2}}\right)\left\{k_{0}^{2}\left(n_{10}^{2}-n_{20}^{2}\right)-\frac{g H_{0}^{2}}{2}\right\}, \\
d_{\varphi}=\frac{\mu^{2}}{4}\left(1+\operatorname{tg}^{2} \varphi\right)+b_{\varphi}, \\
D_{\varphi}=\left\{\delta \varepsilon_{\varphi} d_{\varphi}-\left(\frac{\mu \varepsilon_{1}}{\varepsilon_{2}}\right)^{2}\right\}^{2}-4 \delta \varepsilon_{\varphi}^{2}\left\{d_{\varphi}^{2}\left(\frac{\mu \varepsilon_{1}}{\varepsilon_{2}}\right)^{2} b_{\varphi}\right\},
\end{gathered}
$$

При $\varphi=0$ из (28) можно получить закон дисперсии в виде

$$
\beta^{2}\left(H_{0}\right)=n_{20}^{2}+\frac{1}{k_{0}^{2}}\left\{\left(\frac{\mu \varepsilon_{1}}{2 \varepsilon_{2}}\right)^{2}+\frac{g H_{0}^{2}}{2}\right\}
$$

который представляет собой линейную зависимость от квадрата амплитуды поля. Из (28) также следует, что $\operatorname{tg} \varphi<0$.

Если амплитуда на границе раздела кристаллов связана с оптическими характеристиками кристаллов специальным соотношением (получаемым из условия $\left.\left(\mu \varepsilon_{1} / \varepsilon_{2}\right)^{2}=2 \delta \varepsilon_{\varphi} d_{\varphi}\right):$

$$
H_{0}^{2}=\frac{2}{g}\left\{\frac{\mu^{2} \varepsilon_{2}}{2 \varepsilon_{1}}\left(1+\operatorname{tg}^{2} \varphi-\frac{2}{a_{\varphi}}\right)+k_{0}^{2}\left(n_{10}^{2}-n_{20}^{2}\right)\right\},
$$

то из (27) следует зависимость константы распространения поверхностной волны

$$
\beta^{2}\left(H_{0}, \varphi\right)=n_{10}^{2}-\frac{1}{\delta \varepsilon_{\varphi} k_{0}^{2}}\left\{\left(\frac{\mu \varepsilon_{1}}{\varepsilon_{2}}\right)^{2} b_{\varphi}-d_{\varphi}^{2}\right\}^{1 / 2}
$$

куда подставляется (30). Для существования такой поверхностной волны должно выполняться условие $\left(\mu \varepsilon_{1} / \varepsilon_{2}\right)^{2} b_{\varphi}>d_{\varphi}^{2}$.

Если амплитуда на границе раздела кристаллов связана с оптическими характеристиками кристаллов специальным соотношением (получаемым из условия $\left.\left(\mu \varepsilon_{1} / \varepsilon_{2}\right)^{2} b_{\varphi}=d_{\varphi}^{2}\right)$ :

$$
\begin{aligned}
H_{0}^{2}= & \frac{2}{g}\left\{\frac{\mu^{2}}{2}\left(\frac{\varepsilon_{1}}{\varepsilon_{2}}-\frac{\varepsilon_{2}}{2 \varepsilon_{1}}\left(1+\operatorname{tg}^{2} \varphi\right)\right)\right. \\
& \left.+k_{0}^{2}\left(n_{10}^{2}-n_{20}^{2}\right) \pm \sqrt{\left(\frac{\varepsilon_{1}}{\varepsilon_{2}}\right)^{2}-\left(1+\operatorname{tg}^{2} \varphi\right)}\right\},
\end{aligned}
$$

то из (28) следует зависимость константы распространения поверхностной волны

$$
\beta^{2}\left(H_{0}, \varphi\right)=n_{10}^{2}-\frac{1}{k_{0}^{2}}\left\{a_{\varphi} d_{\varphi}-\left(\frac{\mu \varepsilon_{1}}{\varepsilon_{2}}\right)^{2}\right\},
$$

куда подставляется (32). Для существования такой поверхностной волны значения $\varphi$ выбираются из условий: $\left(\mu \varepsilon_{1} / \varepsilon_{2}\right)^{2}<a_{\varphi} d_{\varphi}$ и $\cos \varphi>\varepsilon_{2} / \varepsilon_{1}$.

\section{5. Заключение}

Таким образом, в данной работе установлено, что на границе раздела фоторефрактивного кристалла и керровского кристалла с фокусирующей нелинейностью могут существовать нелинейные поверхностные волны несимметричного профиля двух типов. Волны первого типа затухают при удалении от границы раздела без осцилляций как в глубину фоторефрактивного кристалла, так и керровского, а волны второго типа затухают в глубину фоторефрактивного кристалла с осцилляциями. Волны первого типа могут затухать монотонно при определенных условиях связи константы распространения, коэффициентов преломления и других физических характеристик фоторефрактивного и керровского кристаллов.

Возможность существования волн с осциллирующим затуханием при определенных условиях принципиально отличает контакт фоторефрактивного кристалла с фокусирующей керровской средой от контакта двух нелинейных керровских сред или контакта нелинейной и линейной сред. Данное обстоятельство имеет существенное значение для проектирования различных оптических устройств (переключателей, сенсоров), использующих волноводные свойства нелинейных поверхностных волн [25-27].

\section{Список литературы}

[1] V. Tekkozyan, A. Babajanyan, K. Nerkararyan. Opt Commun. 305, 190 (2013).

[2] Y.V. Bludov, D.A. Smirnova, Yu.S. Kivshar, N.M.R. Peres, M.I. Vasilevsky. Phys. Rev. B 89, 035406 (2014).

[3] I.S. Panyaev, D.G. Sannikov. J. Opt. Soc. Am. B. 33, 220 (2016).

[4] A.I. Ignatov, I.A. Nechepurenko, D.G. Baranov. Ann. Phys. 528, 537 (2016).

[5] D. Yang, Tian H. J. Opt. 18, 1 (2016).

[6] F.I. Haddouche, L. Cherbi. Opt. Commun. 382, 132 (2017).

[7] Y.M. Aleksandrov, V.V. Yatsishen. J. Nano- and Electronic Phys. 9, 03039 (2017).

[8] В.Н. Белый, Н.А. Хило. Письма в ЖТФ 23, 31 (1997).

[9] Д.Х. Усиевич, Б.А. Нурлигареев, В.А. Сычугов, Л.И. Ивлева, П.А. Лыков, Н.В. Богодаев. Квантовая электрон. 40, 437 (2010).

[10] Д.Х. Усиевич, Б.А. Нурлигареев, В.А. Сычугов, Л.И. Ивлева. Квантовая электрон. 43, 14 (2013). 
[11] С.А. Четкин, И.М. Ахмеджанов. Квантовая электрон. 41, 980 (2011).

[12] Yu.S. Kivshar, G.P. Agrawal. Optical Solitons: From Fibers to Photonic Crystals, Academic Press, San Diego (2003). 540 p.

[13] Yu.S. Kivshar, A.M. Kosevich, O.A. Chubykalo. Phys. Rev. A 41, 1677 (1990).

[14] F.Kh. Abdullaev, B.B. Baizakov, B.A. Umarov. Opt. Commun. 156, 341 (1998).

[15] A.A. Sukhorukov, Yu.S. Kivshar. Phys. Rev. Lett. 87, 083901 (2001).

[16] С.Е. Савотченко. Изв. вузов. Физика 47, 79 (2004).

[17] S.E. Savotchenko. Mod. Phys. Lett. B 32, 1850120 (2018).

[18] С.Е. Савотченко. ЖЭТФ 154, 514 (2018).

[19] N.N. Ahmediev, V.I. Korneev, U.V. Kuzmenko. JETP 88, 107 (1985).

[20] С.Е. Савотченко. Конденсированные среды и межфазные границы 19, 567 (2017).

[21] С.Е. Савотченко. Вестн. ВГУ. Сер.: Физика. Математика 1, 44 (2018).

[22] S.E. Savotchenko. Surfaces Interfaces 13, 157 (2018).

[23] М.П. Петров, С.И. Степанов, А.В. Хоменко. Фоторефрактивные кристаллы в когерентной оптике, Наука, Спб. (1992). 320 c.

[24] V.S. Zuev, J. Russ. Laser Res. 26, 347 (2005).

[25] N. Zhong, Z. Wang, M. Chen, X. Xin, R. Wu, Y. Cen, Y. Li. Sensors Actuators B 254, 133 (2018).

[26] D. Zhang, Z. Li, W. Hu, B. Cheng. Appl. Phys. Lett. 67, 2431 (1995).

[27] T. Strudley, R. Bruck, B. Mills, O.L. Muskens. Light: Science \& Applications 3, e207 (2014).

Редактор Т.Н. Василевская 